\title{
UTILIZAÇÃO DE DADOS CENSITÁRIO PARA A ANÁLISE DE POPULAÇÃO EM ÁREAS DE RISCO
}

\author{
Daiane Batista de Souza; Pilar Amadeu de Souza; Júlia Vicente Martins Ribeiro; Rodrigo \\ Amorim Souza de Moraes Santana; Mariane Carvalho de Assis Dias; Silvia Midori Saito; \\ Regina Célia dos Santos Alvalá.
}

Resumo

Os desastres de origem hidrometeorológica, como deslizamentos e inundações e seus efeitos sobre a população em risco tornaram-se um assunto relevante. O presente artigo tem como objetivo explorar as potencialidades da metodologia desenvolvida em parceria entre o IBGE e o CEMADEN, que delimitou novas áreas para associação de dados demográficos às áreas de risco, denominadas BATER (Base Territorial Estatística de Risco), cujos resultados foram publicados em 2018. A metodologia foi orientada pelo desafio de associar diferentes geometrias de representação, áreas de risco e dados espaciais do Censo Demográfico de 2010. Dos 872 municípios brasileiros monitorados, estima-se que 8.270.127 habitantes e 2.471.349 domicílios privados permanentemente ocupados estavam localizadas em áreas de risco. Por fim, considerou-se que a BATER junto com as pesquisas provenientes de dados do censo podem fornecer informações relevantes para análises intraurbanas sobre a população exposta ao risco de desastres no Brasil.

Palavras-chave: BATER, dados censitários, desastres, vulnerabilidade

\begin{abstract}
The disasters from hidrometeorological origin, such as landslides and floodings and its effects on at-risk population has become a relevant subject. The present article aims to explore the potential of the methodology developed between IBGE and CEMADEN, wich delimited new areas for the association of demographic data to risk areas, called BATER (Statistical Territorial Base of Risk), whose results were published in 2018. The methodology was guided by the challenge of associating different representation geometries, risk areas and spatialized data of the 2010 Demographic Census. Among 872 Brazilian municipalities monitored, it was estimated that $8,270,127$ inhabitants and $2,471,349$ permanent occupied housing units were in risk areas. Finally, it was considered the BATER together as the census data survey, can provide information relevant to intraurban analysis on the population exposed to the risk of disasters in Brazil.
\end{abstract}

Key words: BATER, census data, disasters, vulnerability 
aproximadamente, 170 milhões em 2000. Em 1970 o país tornou-se predominantemente urbano (IBGE, 2007). Em 2010 quase 84\% da população brasileira vivia em áreas urbanas (IBGE, 2013).

As características físicas e topográficas dos terrenos brasileiros não foram um fator limitante para esse intenso processo de ocupação, sobretudo aquele que ocorreu nas regiões dos planaltos e serras do Atlântico Oeste-Sudeste, compostas principalmente por morros com formas de topos convexos, elevada densidade de canais de drenagem e vales profundos, também conhecidos como mares de morros, e das planícies e tabuleiros litorâneos, ao longo da costa leste do país (ROSS, 2005; AB'SABER, 1970). Se por um lado a temática de habitação do espaço urbano na agenda política brasileira teve destaque a partir da década de 1930, quando principalmente os serviços de saneamento básico, como provimento de água e tratamento de esgoto foram privilegiados visando, sobretudo a redução da taxa de mortalidade da população (IBGE, 2007), por outro lado as medidas legais de planejamento urbano não se mostraram eficientes para evitar a ocupação de terrenos não aptos e de forma irregular.

A paisagem urbana no Brasil reflete as desigualdades sociais agudas e necessita de uma reflexão sobre o tema. O rápido processo de urbanização ocorreu junto à expropriação da terra no campo, o que levou uma massa de trabalhadores rurais a ocupar áreas suscetíveis a inundações e deslizamentos (RIBEIRO, 2010). Como resultados, decorreram problemas ambientais envolvendo a destinação do lixo, o abastecimento de água e o acesso ao saneamento básico, presentes no espaço urbano (PORTO-GONÇALVES, 2006).

Esse cenário de crescente urbanização resultou na ocupação de áreas localizadas em encostas íngremes e margens de corpos d'água. Associado a este contexto de ocupação, destacase também os altos volumes pluviométricos registrados nessas regiões que por vezes ocorrem com intensidade moderada a forte (CAVALCANTI et al, 2009). Esta situação combinada com as condições de ocupação e exposição no território pode deflagrar desastres de origem hidrometeorológica, como movimentos de massa (inclui-se, por exemplo, deslizamentos, corridas de lama, de detrito, entre outros), inundações, enchentes, enxurradas e alagamentos.

Nas últimas décadas têm crescido o registro de ocorrência desses desastres no Brasil, sobretudo de movimentos de massa. Embora eventos relacionados à ausência de chuva, como a estiagem e a seca, concentrem o maior número de afetados por desastres, os movimentos de massa e enxurradas são responsáveis pelo maior número de vítimas fatais (CEPED, 2012).

Diante da atual situação da temática de desastres no Brasil, com o aumento constante das ocorrências no espaço urbano, se faz urgente o desenvolvimento de metodologias que visem conhecer as áreas de risco assim como a população em risco e suas condições de vida no território urbano. Se por um lado o conhecimento das áreas de risco do país passou da casa de dezenas para centenas de mapeamentos realizados ao longo dos últimos anos, por outro lado, o mesmo não 
ocorreu visando conhecer detalhadamente a população exposta nessas áreas. Conhecer a população em risco é uma das premissas para um eficiente plano de gestão e resposta a desastres que vise prioritariamente ações de prevenção e salvaguarda da vida humana (Assis Dias et al, 2017).

Com isso, o Centro Nacional de Monitoramento e Alertas de Desastres Naturais (CEMADEN), responsável pelo monitoramento das áreas de risco de movimentos de massa e inundação de municípios críticos do Brasil uniu esforços com o Instituto Brasileiro de Geografia e Estatística (IBGE), principal provedor de informações demográficas no país. No escopo da parceria, objetivou-se o desenvolvimento de pesquisas aplicadas e a geração de bases de dados e informações associadas à caracterização das populações vulneráveis a desastres em áreas urbanas do território brasileiro (IBGE \& CEMADEN, 2018).

Um dos principais produtos da parceria foi a geração e a disponibilização de uma base de dados que dispõe de variáveis capazes de descrever a população exposta em áreas de risco no Brasil. Esta base foi gerada a partir da associação de dados populacionais coletados no censo demográfico de 2010 às áreas de risco mapeadas nos últimos anos. Essa metodologia foi inicialmente testada em três municípios da região serrana do Rio de Janeiro (ASSIS DIAS et al, 2018). Diante da validação dos resultados, a metodologia foi replicada em 872 municípios brasileiros monitorados pelo CEMADEN (IBGE \& CEMADEN, 2018). No presente artigo apresenta-se a metodologia desenvolvida para a criação da base de dados de população em áreas de risco de desastres, bem como suas potencialidades de uso e aplicação.

\section{Risco e vulnerabilidade da população a desastres}

Em âmbito nacional, a quantificação dos registros de ocorrência de desastres relacionados a ameaças naturais cresceu significativamente, sendo comprovada pela série histórica do Atlas Brasileiro de Desastres Naturais, organizada pelo CEPED (UFSC). Os dados desta publicação evidenciaram que o maior número de afetados, entre os anos de 1991 e 2012, estava associado a estiagem e seca, seguido de enxurrada e inundação. O maior número de vítimas fatais foi decorrente dos movimentos de massa e inundações (CEPED, 2013).

Nesse contexto, de acordo com Tominaga (2011), os desastres predominantes no Brasil são os derivados da dinâmica externa da Terra, isto é, os deslizamentos de solos e rochas, as inundações e enchentes e as tempestades. A autora ainda analisou a tipologia dos desastres mais frequentemente atendidos pela Defesa Civil, de acordo com as regiões geográficas do país: presença predominante de incêndios florestais e inundações na Região Norte; secas e inundações 
na Região Nordeste; incêndios florestais na Região Centro-Oeste; deslizamentos e inundações na Região Sudeste; e inundações, vendavais e granizos, na Região Sul.

Ao longo do tempo a compreensão a respeito da temática de desastres, tem sido discutida sob diversos pontos de vistas. Nosso entendimento condiz com a UNISDR (2017, tradução nossa) ${ }^{1}$ , que conceituam os desastres como uma "séria interrupção do funcionamento de uma comunidade ou de uma sociedade em qualquer escala devido a eventos perigosos que interagem com condições de exposição, vulnerabilidade e capacidade, levando a uma ou mais das seguintes: perdas e impactos humanos, materiais, econômicos e ambientais".

Destaca-se, ainda, nesse contexto, que os riscos de desastre estão associados à interação entre as forças da natureza, porção da superfície terrestre e suas características singulares, com um sistema social potencialmente vulnerável a estas forças. Sendo assim, pode-se dizer que só existe risco caso ele seja atuante em um espaço ao homem e ao meio ambiente, induzindo a perdas de vida, ferimentos ou bens destruídos em uma sociedade. O risco pode ser determinado, probabilisticamente, em função da ameaça, exposição e vulnerabilidade (CUNHA E DIMUCCIO, 2002; TRAJBER, 2014a; TRAJBER, 2014b; UNISDR, 2017).

A partir do exposto, para compreender os aspectos que colocam a população mais ou menos exposta a ser afetada por um evento de desastre, faz-se uso do conceito de vulnerabilidade com um enfoque multidisciplinar, englobando não somente as características estruturais, mas também as sociais.

Na concepção da UNISDR (2017, tradução nossa) ${ }^{2}$, vulnerabilidade se refere às "condições determinadas por fatores ou processos físicos, sociais, econômicos e ambientais que aumentam a suscetibilidade de um indivíduo, comunidades, bens ou sistemas aos impactos de ameaças". Nesse sentido, uma única ameaça pode causar efeitos distintos a diferentes partes de um sistema, dependendo dos elementos que o constituem, i.e. o padrão construtivo das estruturas que estão expostas ao risco e as características socioeconômicas de uma população. Assim, é um conceito social resultante da combinação de processos econômicos, sociais, ambientais e políticos. Remete ao grau de preparo de um determinado grupo social frente às ameaças, seja por questões de resiliência, renda; qualificação das pessoas residentes naquela sociedade; seja pelas condições de saúde física e mental; pelas construções ou pela estrutura política e institucional (LAVELL, 2000). Outras características também interferem na questão da vulnerabilidade, como condições de moradia (esgotamento sanitário, destinação do lixo, abastecimento de água); e a caracterização da

\footnotetext{
1 "A serious disruption of the functioning of a community or a society at any scale due to hazardous events interacting with conditions of exposure, vulnerability and capacity, leading to one or more of the following: human, material, economic and environmental losses and impacts".

2 "The conditions determined by physical, social, economic and environmental factors or processes which increase the susceptibility of an individual, a community, assets or systems to the impacts of hazards."
} 
população, como identificação das faixas etárias mais vulneráveis, educação, gênero, entre outros aspectos (GARCIA-TORNEL, 1997; LEONE, 2004; AVISO, 2005).

Nessa medida, ressalta-se a importância de conhecer as características socioeconômicas de populações que residem em áreas de risco a desastres. Para isso é imprescindível o desenvolvimento de metodologias e geração de bases de dados sólidas que permita subsidiar análises integradas de risco de desastres no país.

\section{Procedimentos Metodológicos}

Frente a urgência de identificar as características da população habitante em áreas de risco para subsidiar ações de prevenção e monitoramento de desastres, optou-se por utilizar os dados populacionais coletados no censo demográfico de 2010 (IBGE).

Os mapeamentos de áreas de risco de deslizamentos, inundações e enxurradas, utilizados nessa base, foram realizados por diversas instituições brasileiras, tais como, a Companhia de Pesquisas e Recursos Minerais - CPRM, as Prefeituras Municipais através dos Planos Municipais de Redução de Riscos - PMRRs, o Departamento de Recursos Minerais - DRM do Rio de Janeiro, o Instituto de Pesquisas Tecnológicas - IPT e o Instituto Geológico - IG.

A análise da distribuição espacial dos setores censitários e das áreas de risco evidenciou que, em muitos casos, as áreas de risco pertencem a um ou mais setores censitários. Isso ocorre, pois, as bases de dados foram criadas com objetivos diferentes, uma para coleta de informações populacionais e outra para determinar os limites das áreas de risco associadas a um fenômeno perigoso (ASSIS DIAS et al, 2017). Devido às diferenças nas formas de representação dos dados geográficos oriundos dos mapeamentos das áreas de risco e dos recortes territoriais do censo demográfico, verificou-se o desafio de integrar as diferentes geometrias das informações geográficas em uma única base de dados.

No intuito de solucionar esse desafio metodológico, os dados foram processados em um Sistema de Informações Geográficas (SIG), porém a complexidade da compatibilização das diversas escalas e formatos dos dados acima citados não possibilitou a utilização de procedimentos automáticos. Assim foi necessário contar com a experiência de técnicos de geoprocessamento habilitados a interpretar e delimitar manualmente as unidades espaciais, para fazer coincidir em um único recorte territorial setores censitários e área de risco. Como resultado foi criada a Base Territorial Estatística de Áreas de Risco (BATER), que foi disponibilizada publicamente em 2018 pelo IBGE em sua plataforma digital ${ }^{3}$.

3 Disponível em: <https://www.ibge.gov.br/apps/populacaoareasderisco/>. 
Dentre as mais de 600 variáveis coletadas no censo (por moradores e por domicílios), foram elencadas 183 mais representativas para caracterização da população e 135 para domicílio. Desse conjunto, foram selecionadas as variáveis número de moradores por domicílio, sexo, faixa etária e renda para população; e acesso a serviços básicos, como abastecimento de água, esgotamento sanitário, coleta de lixo e energia elétrica para os domicílios como variáveis relevantes para a caracterização de população em área de risco ${ }^{4}$.

A periodicidade e a experiência do IBGE em suas coletas permite acompanhar as variações do contingente populacional e da situação das moradias, possibilitando a realização de análises temporais nas referidas áreas, em todos os municípios monitorados pelo CEMADEN espalhados pelo território brasileiro, garantindo a abrangência da pesquisa a nível nacional e possibilitando identificar a situação de cada município com objetivo de direcionar investimentos em políticas públicas e obras de contenção. Ou seja, sendo o censo demográfico aplicado em todos os 5.570 municípios brasileiros, a presente metodologia pode ser replicada para todos os municípios que disponham de mapeamentos de áreas de risco.

\section{Breve panorama sobre a população exposta a desastres no Brasil}

A associação das informações oriundas do censo demográfico e dos mapeamentos de áreas de risco de desastres no Brasil, a partir da utilização de ferramentas de Geoprocessamento proporcionou a obtenção de informações específicas sobre a distribuição espacial e características da população exposta e vulnerável em áreas de risco de desastre em escala nacional (IBGE e CEMADEN, 2018).

Diante dessas informações geográficas analisadas e já divulgadas pelo recorte da BATER para 872 municípios, foi estimado que 8.270.127 habitantes e 2.471 .349 domicílios particulares permanentes se encontravam em áreas de risco de desastres em 2010. Desse total, 17,8\% dos habitantes pertenciam ao grupo etário mais vulnerável, composto por 9,2\% de crianças com idade menor de 5 anos e 8,5\% de idosos com idade superior a 60 anos. Também foi contabilizado que somente $6,5 \%$ desses moradores expostos apresentavam domicílios sem cobertura de abastecimento de água por rede geral; 26,1\% não dispunham de acesso à rede geral de esgoto ou fossa séptica, formas estas de esgotamento consideradas adequadas; e 4,1\% dessas pessoas em áreas de risco não possuíam destinação de lixo adequado.

Para a realização deste trabalho, foram selecionadas 27.660 áreas de risco que correspondem àquelas situadas em municípios monitorados pelo CEMADEN, 197.649 feições

\footnotetext{
4 Todas as variáveis elencadas por moradores e domicílios estão disponibilizadas na publicação de População em áreas de risco no Brasil. Disponível em: <https://www.ibge.gov.br/apps/populacaoareasderisco/>.
} 
censitárias obtidas pelo IBGE, sendo que 2,16\% (4.272) foram providas por setores censitários urbanos, e 97,84\% (193.377) foram originadas por faces de quadra, formando 8.309 polígonos da Base Territorial Estatística de Áreas de Risco.

A variável que foi selecionada, nesta publicação, como mais relevante para a compreensão da vulnerabilidade dos moradores em risco, foi a de faixa etária. Crianças e idosos são considerados como os mais vulneráveis a desastres por necessitarem de cuidados especiais, em razão da maior dependência para locomoção, por permanecerem mais tempo em seus domicílios e apresentarem menor capacidade de resistir a ferimentos (WISNER et al., 2003). Nas condições de domicílios as variáveis que foram levadas em consideração, são referentes às formas de abastecimento de água, ao esgotamento sanitário e à destinação do lixo. Estes serviços utilizados de forma inadequada além de revelarem a precariedade das condições dos domicílios, induzem ao favorecimento da concentração de fluxos nas encostas, potencializam o aumento da saturação do terreno e intensificam processos de inundações e deslizamentos (ARMESTO, 2012; MIRANDOLA e MACEDO, 2014), como também podem aumentar a exposição da população ao risco de desastres.

$\mathrm{Na}$ análise regional dos dados, a Região Sudeste destacou-se na quantidade de áreas de risco, distribuídas em 308 dos seus municípios monitorados e também na contagem populacional residindo nessas áreas, com 4.266.301 (9,8\% da população total dos municípios monitorados da região). Contabilizou-se que $4,5 \%$ dessa população em áreas de risco não contava com abastecimento de água por rede geral; 17,7\% viviam em domicílios com esgotamentos sanitários inadequados; e apresentavam valores inferiores a 3,0\% de moradores sem destinação adequada de lixo: $1,99 \%$.

A Região Nordeste apresentou 294 municípios monitorados com 2.952.628 pessoas vulneráveis a deslizamentos e inundações $(11,4 \%$ da população total dos seus municípios monitorados), em 2010. Além disso, foi contabilizado que esta população em risco apresentava 9,1\% de indivíduos com idade menor de 5 anos e 8,4\% de indivíduos com idade superior a 60 anos; 6,7\% não possuía abastecimento de água por rede geral; 34,5\% dos moradores em áreas de risco possuíam esgotamento sanitário inadequado; e 6,6\% desta população não apresentava destinação adequada de lixo.

A Região Sul, apresentava 703.368 pessoas vivendo em áreas de risco, nos 144 municípios monitorados (6,0\% da população total dos seus municípios monitorados). Foi contabilizado que esta população exposta aos riscos era composta por $8,7 \%$ de pessoas com idade inferior a 5 anos e $9,7 \%$ de pessoas com idade superior a 60 anos; 8,5\% desses habitantes não possuíam abastecimento de água por rede geral; 19,7\% dessas pessoas residiam em domicílios sem acesso a esgotamento sanitário adequado; e percentual inferiores a 3,0\% de moradores nessas condições de risco não apresentavam destinação adequada de lixo: 1,49\%. 
A Região Norte continha 107 municípios monitorados com 340.204 de moradores residindo em áreas de risco (3,9\% da população total de seus municípios monitorados). Foi registrado que 13,0\% da população vulnerável era composta por crianças com menos de 5 anos de idade e 6,0\% por idosos com idade superior a 60 anos; $26,3 \%$ não apresentavam abastecimento de água por rede geral; $70,7 \%$ dos moradores em áreas de risco apresentavam inadequação das condições de esgotamento sanitário; e 14,1\% de moradores em risco sem destinação adequada de lixo. Esta Região com maior número de crianças expostas vivendo em áreas de risco e domicílios com coleta de lixo irregular.

A Região Centro-Oeste registrou a menor quantidade de áreas de risco e consequentemente, de população nessa condição de risco, isto é, 7.616 moradores $(0,3 \%$ da população total dos seus 19 municípios monitorados na região). Contabilizou que 9,7\% de crianças com idade menor de 5 anos e 8,4\% de idosos com idade superior a 60 anos; 3,9\% não possuía abastecimento de água por rede geral; $52,5 \%$ pessoas habitavam moradias com esgotamento sanitário inadequado; e apresentaram valores inferiores a 3,0\% de moradores sem destinação adequada de lixo: $2,43 \%$.

\section{Potencialidades dos dados da BATER enquanto recorte territorial}

\section{Índices de vulnerabilidade}

Existem diferenças na conceituação da vulnerabilidade e aplicação nas mais diversas áreas, entretanto, na maioria desses conceitos há um ponto de convergência: a vulnerabilidade significa potencial de perda diante da ocorrência de um desastre (CUTTER et al, 2003). Nesse sentido, destaca-se a importância de definir qual o tipo de perda e de quem são essas perdas, ou seja, o que está exposto e quem são as pessoas expostas. Saito (2011) aponta que além das perdas materiais e dos moradores expostos, também é essencial identificar as condições de vida para se restabelecer após a ocorrência de um desastre. Indicadores de exposição e capacidade de resposta são, portanto, essenciais em estudos de vulnerabilidade social a desastres. Cutter et al (2003) levantou os indicadores de vulnerabilidade social mais frequentemente encontrados na literatura e identificou que algumas características individuais são amplamente utilizadas, como idade, gênero, raça e rendimento. Por outro lado, aspectos da comunidade podem também impactar as respostas individuais, como nível de urbanização e taxas de desenvolvimento econômico a que determinados grupos estão expostos.

Especificamente no Brasil, alguns esforços já foram realizados no intuito de analisar vulnerabilidade em diferentes contextos, não necessariamente associados a desastres, tendo 
principalmente como referência a escala municipal ou ao nível de setores censitários. Nesse sentido, a escala utilizada no presente trabalho (proveniente de informações da face de quadra e de setores censitários) é um diferencial e permite a utilização e combinação das variáveis que descrevem as condições de vida dos moradores e as condições de moradia no território intraurbano, podendo, desta forma, indicar as vulnerabilidades da população residente (SEADE, 2010; COSTA; MARGUTTI, 2015; ALMEIDA et al, 2016).

Uma das premissas de estudos de vulnerabilidade é que a mesma varia no tempo e no espaço. Se, por um lado, a atual base de dados permite análises espaciais intraurbanas e com ganho de informação inédita, por outro, conforme ocorram os novos censos demográficos brasileiros, a geração das novas bases de dados pode permitir a análise temporal. A aplicação de análises espaciais e temporais integradas pode ajudar a responder questões norteadoras desse tipo de estudo, se de fato as sociedades estão se tornando mais ou menos vulneráveis aos desastres (CUTTER, 1996).

\section{Ampliação e refinamento da pesquisa}

Conforme IBGE (IBGE, 2019) "um território deve ser analisado em sua pluralidade, isto é, sob inúmeras perspectivas, escalas e ângulos de análise". Nesse sentido, a incorporação de outras pesquisas, sejam voltadas para questões populacionais, econômicas ou territoriais, tem como intuito dar conta da complexidade envolvida na questão das áreas de risco e sua população.

As pesquisas intraurbanas desenvolvidas no IBGE oferecem elementos importantes para definição de populações em risco como o estudo de Tipologia Intraurbana (IBGE, 2017) baseado nos dados do censo demográfico 2010 e contribui para este fim, na medida em que detalha a diversidade socioeconômica e de infraesturtura nas áreas residenciais presentes nas Concentrações Urbanas brasileiras, acima de 300 mil pessoas (IBGE, 2017). Essa pesquisa, ao classificar a população urbana em 11 categorias, de acordo com as condições das moradias e aspectos socioeconômicos da população, auxilia o refinamento na classificação das áreas de risco.

Outro produto do IBGE importante para a compreensão do espaço urbano é o conceito de Aglomerados Subnormais, que norteia a delimitação dos diversos tipos de assentamentos irregulares existentes no país. A junção desses assentamentos com a base BATER permitirá a identificação de áreas de risco que coincidem com os limites dos aglomerados subnormais. Identificando, assim, os espaços urbanos com no mínimo 51 unidades habitacionais que, além de estarem submetidos a riscos de desastres, caracterizam-se pela ausência de titularidade da propriedade, carência de serviços públicos básicos, padrão urbanístico irregular e localização em áreas que apresentam restrições legais à ocupação (IBGE, 2010). A pesquisa Aglomerados 
Subnormais oferece um recorte territorial urbano que deve ser visto com atenção especial pela pesquisa das populações em áreas de risco, na medida em que identifica populações de baixa renda assentada de forma irregular em sítios urbanos que não receberam infraestrutura adequada para moradias.

Outra pesquisa do IBGE que está intrinsecamente relacionada às questões urbanas e também pode ser do interesse para diagnóstico de áreas de risco é a Pesquisa Territorial do Entorno Urbanístico dos Domicílios, incorporada ao questionário do censo 2020. Essa pesquisa levanta informações sobre infraestrutura e pavimentação da via, iluminação pública; infraestrutura de transporte; acessibilidade urbana; escoamento pluvial e arborização. Desta forma, a inclusão de elementos físicos ou naturais que descrevem o ambiente no qual vive a população exposta, viabiliza o conhecimento detalhado do sítio em que a população está assentada. Permite, também, elaborar ações eficientes para diferentes fases da gestão de risco de desastres, como refinar ainda mais as metodologias de índices de vulnerabilidade socioambiental, em uma escala intraurbana e entender as diferentes respostas aos alertas, dadas às capacidades estruturais do ambiente (IBGE, 2010).

\section{Considerações Finais}

O presente trabalho apresentou a importância das pesquisas desenvolvidas no IBGE principalmente aquelas inseridas no censo, para subsidiar a construção da pesquisa População em áreas de risco no Brasil.

A metodologia associou informações de bases de dados nacionais já coletadas para outros fins, como os mapeamentos de áreas de risco e dados coletados no censo demográfico brasileiro logo, a base de dados gerada é o resultado de um esforço interinstitucional, i.e. IBGE e CEMADEN, e evidencia a multiplicação no aproveitamento de recursos públicos já empregados.

Dentre as vantagens da metodologia proposta destaca-se sua aplicação para qualquer município brasileiro que disponha de mapeamento de áreas de risco, já que o censo demográfico abrange todos os municípios do país, em toda sua extensão habitada. Outro ponto central da metodologia é a possibilidade de atualização das informações da população exposta, conforme ocorram atualizações dos mapeamentos de risco bem como novos censos demográficos. Também, é possível refinar a pesquisa a partir de outras pesquisas do IBGE como, Tipologia Intraurbana (IBGE, 2017), Aglomerados Subnormais (IBGE, 2010) e a Pesquisa Territorial do Entorno Urbanístico dos Domicílios (IBGE, 2010).

Ainda que originalmente a base tenha sido desenvolvida para atender o sistema de monitoramento e alerta do CEMADEN, é evidente seu potencial de uso e aplicações em outras áreas do conhecimento. Novas potencialidades são estimuladas a serem desenvolvidas por 
possíveis usuários para que se possa avaliar a base de dados produzida e, eventualmente, implementar melhorias para a versão que poderá ser produzida a partir de novos censos.

Importante ressaltar que a produção de BATER para outros municípios brasileiros críticos, monitorados pelo CEMADEN, continua em desenvolvimento baseada nos dados do censo demográfico 2010. Os primeiros esforços concentraram-se em aplicar a metodologia desenvolvida para 872 municípios brasileiros monitorados pelo CEMADEN. Conforme já exposto, a aplicação da metodologia é perfeitamente possível para novos censos. Assim, o acompanhamento temporal da exposição da população em áreas de risco no país poderá ser realizado com a periodicidade de 10 anos. Dessa maneira, é notável a intrínseca relação entre a metodologia criada e as informações provenientes de novos censos para a contínua identificação da população e de suas condições de exposição a desastres no Brasil.

Agradecimentos

Os autores Daiane Batista de Souza; Pilar Amadeu de Souza; Júlia Vicente Martins Ribeiro; Rodrigo Amorim Souza de Moraes Santana; Mariane Carvalho de Assis Dias agradecem ao Conselho Nacional de Desenvolvimento Científico e Tecnológico (CNPq) pelo apoio financeiro. As autoras Silvia Midori Saito eRegina Célia dos Santos Alvalá agradecem ao INCT Climate Change Projeto Fase 2 (Processo 465501014-1/ Chamada Pública MCTI /CNPQ /CAPES /FAPESP No 16/2014). Este artigo é uma contribuição da Rede Brasileira de Pesquisa sobre Mudanças Climáticas Globais FINEP / 
Referências

AB'SABER, A. Domínios morfoclimáticos e províncias fitogeográficas do Brasil. Revista Orientação, São Paulo: IG-USP, 1970.

ALMEIDA, L.Q.; WELLE, T.; BIRKMANN, J. Disaster risk indicators in Brazil: a proposal based on the world risk index. International Journal of Disaster Risk Reduction. Vol. 17: 251-272, 2016.

ARMESTO, R. C. G. Temas geológicos para educação ambiental, caderno IV - ação da água das chuvas no planeta Terra Parte II, 2012. (Acesso em: setembro, 2017).

ASSIS DIAS, M. C.; SAITO, S. M.; FONSECA, M.R.S. Aplicação de dados censitários para caracterização da população exposta em áreas de risco de deslizamentos em Blumenau, Santa Catarina. Revista Brasileira de Cartografia. № 69/1. Edição Especial Geotecnologias e Desastres Naturais: 193-207, 2017.

ASSIS DIAS, M. C.; SAITO, S. M.; ALVALÁ, R. C.S.; STENNER, C.; PINHO, G.; NOBRE, C. A.; LIMA, C. O. Estimation of exposed population to landslides and floods risk areas in Brazil, on an intra-urban scale. International Journal of Disaster Risk Reduction. Vol. 31: 449-459, 2018.

AVISO. Information Bulletin on Global Environmental Change and Human Security. № 14 , Oct 2005.

CAVALCANTI, I.F.A.; SILVA DIAS, M.A.F.; SILVA, M.G.A.J.; FERREIRA, N.J. (orgs). Tempo e clima no Brasil. São Paulo: Oficina de Textos, Brasil, 2009. 464p.

CEPED - Centro Universitário de Estudos e Pesquisas sobre Desastres, Universidade Federal de Santa Catarina. Atlas Brasileiro de Desastres Naturais: 1991-2010. Vol. Brasil. Florianópolis: CAD UFSC, 2012.

CEPED - Atlas Brasileiro de Desastres Naturais: 1991 a 2012 / Centro Universitário de Estudos e Pesquisas sobre Desastres. Universidade Federal de Santa Catarina 2. ed. rev. ampl. Florianópolis: 2013. 126 p.

COSTA, M. A.; MARGUTTI, B.O. Atlas da vulnerabilidade social nos municípios brasileiros. Brasília: IPEA: 2015.

CUNHA, L. E DIMUCCIO, L.. "Considerações sobre riscos naturais num espaço de transição. Exercícios cartográficos numa área a sul de Coimbra". Territorium, Coimbra, 9, p. 37-51, 2002.

CUTTER, S. Vulnerability to environmental hazards. Progress in Human Geography. Vol. 20: 529539, 1996. 
CUTTER, S.; BORUFF, J.B.; LYNN SHIRLEY, W. Social vulnerability to environmental hazards. Social Science Quarterly, vol. 84, num. 2, 2003.

GARCÍA-TORNEL, F.C. Algunas Cuestiones Sobre Geografía De Los Riesgos. Scripta Nova. Revista Electrónica de Geografía y Ciencias Sociales. Universidad de Barcelona.vol. 1, n. 10 , 15 de nov. 1997, 11 p. Disponível em: <http://www.ub.es/geocrit/sn-60.html>. (Acesso: Junho, 2019).

IBGE (Instituto Brasileiro de Geografia e Estatística). Estatísticas do século XX. Rio de Janeiro. 2007.

IBGE (Instituto Brasileiro de Geografia e Estatística). Aglomerados Subnormais, Primeiros Resultados. Rio de Janeiro. 2010.

IBGE (Instituto Brasileiro de Geografia e Estatística). Características Urbanísticas do Entorno dos Domicílios. Rio de Janeiro. 2010.

IBGE (Instituto Brasileiro de Geografia e Estatística). Atlas do Censo Demográfico 2010. Rio de Janeiro. 2013.

IBGE (Instituto Brasileiro de Geografia e Estatística). Tipologia Intraurbana. https://www.ibge.gov.br/apps/tipologia_intraurbana/ . Rio de Janeiro. 2017. (Acesso: julho, 2019).

IBGE (Instituto Brasileiro de Geografia e Estatística) e CEMADEN (Centro Nacional de Monitoramento e Alertas de Desastres Naturais). População em áreas de risco no Brasil. https://www.ibge.gov.br/apps/populacaoareasderisco/. 2018. (Acesso: junho, 2019).

IBGE (Instituto Brasileiro de Geografia e Estatística). Quadro Geográfico de Referência para Produção, Análise e Disseminação de Estatísticas. Rio de Janeiro. 2019. (Acesso: julho, 2019).

LAVELL, A. Degradación ambiental, riesgo y desastre urbano. Problemas y conceptos: hacia la definición de uma agenda de investigación. In: FERNÁNDEZ, M. A. Ciudades en riesgo. Degradación ambiental, riegos urbanos e desastres en América Latina. Disponível em: http:www.lared.org.pe. 2000. (Acesso: junho, 2019).

LEONE, F. Une approche quantitative de la cartographie dês risques naturels: application expérimentale au patrimoine bati de la Martinique (Antilles françaises).Géomorphologie relief, processus, environnement. avril-juin. 2004, n², 117-126p.

MIRANDOLA, F. A.; MACEDO, E. S. Proposta de classificação de tecnógeno para uso no mapeamento de áreas de risco de deslizamento. In: Quaternary and Environmental Geosciences. v. $5, \quad$ n. 1 , p. 66-81, 2014 . Disponível em: https://revistas.ufpr.br/abequa/article/view/34764. (Acesso em: feveriro, 2018). 
PORTO-GONÇALVES, C.W. A globalização da natureza e a natureza da globalização. Civilização Brasileira. Rio de Janeiro. 2006.

RIBEIRO, W.C. Riscos e Vulnerabilidade Urbana no Brasil. Revista Electrónica de Geografia y Ciencias Sociales. Universidade de Barcelona. ISSN: 1138-9788. Vol XIV, núm 331 (65), 2010.

ROSS, J. L.S. Geografia do Brasil. São Paulo: EDUSP, 2005.

SAITO, S.M. Dimensão socioambiental na gestão de risco dos assentamentos precários do maciço do Morro da Cruz, Florianópolis-SC. Tese de doutorado. Universidade Federal de Santa Catarina. 2011. 252 p.

SEADE- Fundação Sistema Estadual de Análise de Dados. Índice Paulista de Vulnerabilidade Social. 〈http://www.seade.gov.br/analises_estudos/indicepaulista-de-vulnerabilidade-social-ipvsversao-2010/). (Acesso: Fevereiro, 2017).

TOMINAGA, L. K.; SANTORO, J.; AMARAL, R. (Orgs). Desastres naturais: conhecer para prevenir. 1.ed., $2^{\mathrm{a}}$ reimpressão. São Paulo: Instituto Geológico. 2011. 196 p.

TRAJBER, R. Estudo analítico sobre as políticas públicas de educação com relação às mudanças climáticas, sustentabilidade e prevenção de desastres no Brasil. Relatório Técnico elaborado no âmbito do Projeto 914BRZ2018. São José dos Campos: Unesco/Cemaden/MCTI, 2014a.

TRAJBER, R. Contribuição para um plano de educação para a redução de impactos de desastres: percepção de riscos, vulnerabilidade socioambiental e construção de uma cultura de sustentabilidade e resiliência. Relatório Técnico elaborado no âmbito do Projeto 914BRZ2018. São José dos Campos: Unesco/Cemaden/MCTI, 2014b.

UNISDR. United Nations Oficce For Disaster Risk Reduction. 2017. Disponível em: https://www.unisdr.org/. (Acesso: junho, 2019).

WISNER, B.; CANNON, T.; DAVIS, I; BLAIKIE, P. At risk: Natural hazards, people's vulnerability and disasters, 2 ed. New York: Routledge, 2003. Disponível em: https://www.ids.ac.uk/publication/at-risk-natural-hazards-peoples-vulnerability-anddisasters. (Acesso em: fevereiro, 2018). 